\section{THU0076 ADENOVIRUS MEDIATED GENE TRANSFER OF UROKINASE PLASMINOGEN INHIBITOR INHIBITS ANGIOGENESIS IN EXPERIMENTAL ARTHRITIS}

C Jorgensen, F Apparailly, C Bouquet, V Millet, D Noel, P Yeh, J Sany. ImmunoRheumatology, Lapeyronie, Montpellier, France

\subsection{6/annrheumdis-2001.953}

Background Plasmin is essential for metalloproteases (MMP) activation, endothelial cell migration and degradation of the extracellular matrix. The process is common to neoangiogenesis and pannus formation, and $80 \%$ of synovial cells express urokinase plasminogen activator receptor (UPAR).

Objectives We inhibited plasmin activation by expressing uPA/ uPAR antagonist chimeric molecule (ATF-HSA) through adenoviral mediated gene transfer in experimental arthritis.

Methods Overexpression of ATF-HSA was obtained by IV injection of 109 pfu of a non replicative E1 deleted type 5 adenoviral vector containing murine ATF gene linked to HSA gene under the control of a CMV promoter. Collagen-induced arthritis (CIA) was obtained by immunisation of DBA1 mice with bovine type II collagen in FCA. AdATF-HSA was injected IV on day 25 after immunisation. On day 44, X ray and Immuno-histology of paws were performed. The SCID/hu model was used to assess the effect of adenoviral mediated huATF gene transfer on human rheumatoid synovium angiogenesis.

Results

Conclusion The uPAR antagonist ATF-HSA is a new tool for gene therapy in arthritis. The preventive effect observed may be related to the decrease in angiogenesis rather than inhibition of extracellular matrix degradation.

\section{THU0077 INTERATIONS BETWEEN INFLAMMATORY POLYARTHRITIS AND CARDIOVASCULAR DISEASE}

${ }^{1} \mathrm{PH}$ Dessein, ${ }^{1} \mathrm{AE}$ Stanwix, ${ }^{2} \mathrm{BI}$ Joffe, ${ }^{3} \mathrm{EA}$ Shipton. ${ }^{1}$ Rheumatology; ${ }^{2}$ Carbohydrate and Lipid Metabolism Research Unit; ${ }^{3}$ Pain Relief and Research Unit, CH Baragwanath Hospital, Johannesburg, South Africa

\subsection{6/annrheumdis-2001.954}

Background Inflammatory polyarthritis (IP) and cardiovascular disease (CVD) may share a common predisposition and disease activity in IP may be complicated by worsening of CVD risk factors profiles.

Objectives The aim of the present study was to provide evidence for these hypotheses.

Methods A literature search on predisposing factors for IP and a case controlled study in 87 IP patients (38 rheumatoid arthritis, 29 spondyloarthropathy and 20 undifferentiated inflammatory arthritis) on CVD risk factors were performed.

Results Recently identified predisposing factors for IP bear remarkable similarities to CVD risk factors. They include ageing, obesity, smoking, elevated cholesterol and apolipoprotein (apo) (a) concentrations, subtle hypoadrenalism, decreased unsaturated fat intake and antioxidant concentrations, no alcohol intake, infections and stressful life events. Indeed, activation of nuclear factor kappa B, monoclonal expansion of proinflammatory $\mathrm{CD} 4{ }^{+} \mathrm{CD} 28-\mathrm{T}$ cells and acute phase responses (APR), the mutually reinforcing relationship between obesity/insulin resistance (IR) and hypoadrenalism and, endothelial dysfunction may each play a pivotal role in both IP and CVD. In established IP, disease activity associates with the presence of APR, raised apo (a), cholesterol/HDL-cholesterol and apo B/A1 ratios, IR and decreased dehydroepiandrosterone sulphate (DHEAS) concentrations, thereby worsening CVD risk factor profiles. Three studies reported a $60-80 \%$ excess CVD mortality in rheumatoid arthritis. In a more recent investigation, in which disease modifying agents were instituted soon after disease onset, the 10 year survival was similar to the general population. In our controlled study, IP was associated with a positive family history for coronary artery disease $(\mathrm{p}<0.03)$. Twenty five $(29 \%)$ patients had IR and this could be accounted for by the APR and body mass index (BMI). Cholesterol/HDL-cholesterol ratios were elevated and this was only partially attributable to the APR, BMI and IR. Twenty four (28\%) patients had decreased DHEAS concentrations and in women, this could not be fully explained by the APR, IR, disease duration, previous glucocorticoid and current NSAID usage.

Conclusion Further elucidation of etiopathogenetic mechanisms shared by IP and CVD may yield more effective therapies for both conditions. Meanwhile, CVD risk factor profiles should be addressed in IP management.

\section{REFERENCES}

1 Dessein $\mathrm{PH}$, et al. Rheumatology 2001, in press

2 Liuzzo G, et al. Circulation 2000;102: 2883-8

3 Weyand CM, et al. Arch Immunol Ther Exp (Warsz) 2000;48:429-35

4 Kroot EJ, et al. Ann Rheum Dis. 2000;59:954-8

\section{THU0078 ANTIARTHRITIC EFFECT OF SALBUTAMOL ALONE AND IN COMBINATION WITH PENTOXYPHILINE ON ADJUVANT ARTHRITIS IN RATS}

R Bradunaite, E Bernotiene, L Leonaviciene, D Vaitkiene. Laboratory of Experimental Pathology, Institute of Experimental and Clinical Medicine, Vilnius, Lithuania

\subsection{6/annrheumdis-2001.955}

Background The beta-2 ?adrenergic agonist salbutamol (S) and the phosphodiesterase inhibitor pentoxifylline $(\mathrm{P})$ are reported to be the elevators of cAMP and the suppressors of Th1 response. $^{1,2}$ Elevation of intracellular cAMP reduces the production of IL-12 and TNF, the important cytokines in the pathogenesis of rheumathoid arthritis (RA).

Objectives To evaluate the antiarthritic effect of $S$ alone and in combination with $\mathrm{P}$ on the model of $\mathrm{RA}$ in animals - the expressed adjuvant arthritis (AA) in rats.

Methods Experiment was performed on 30 female Lewis rats with AA induced by a single subplantar injection of $0,1 \mathrm{ml}$ of complete Freud's adjuvant into the left hind paw. On day 8 of experiment, rats were assigned into 3 groups with the similar mean scores of joint swelling and the treatment with $S$ (1st group) and $\mathrm{S}+\mathrm{P}$ (2nd) was started. Control group (3rd) received $1 \mathrm{ml}$ of starch gel. Drugs were prepared as the suspension in $1 \%$ starch gel and administered intragastrically 5 times a week in a volume of $1 \mathrm{ml}$ in following doses: $\mathrm{S}$ ? $8,5 \mathrm{mg} / \mathrm{kg}$ and $\mathrm{P}$ ? 500 $\mathrm{mg} / \mathrm{kg}$. The duration of treatment ? 2 weeks. Body weight and joint swelling were monitored 3 times a week. Haematological and pathomorphological changes were evaluated at the end of experiment (day 23).

Results $S$ significantly $(\mathrm{p}<0,02)$ reduced joint swelling by $38,3 \%$ at the end of experiment. Combined treatment with $\mathrm{S}+\mathrm{P}$ was more effective and significantly $(\mathrm{p}<0,02-0,01)$ diminished joint swelling starting at day 18 (by 36\%) till the end of experiment (by 44\%). Polyarthritis developed in $70 \%$ of animals treated with S, $60 \%$ - with S+P and $90 \%$ - of control group. The marked improvement $(\mathrm{p}<0,02)$ of blood indices was 
observed in the both treated groups as compared to the control, but was more expressed using combined therapy (ESR lower by $47,8 \%$ and $56,47 \%$ and the count of leukocytes by $30,8 \%$ and $36,2 \%)$. Toxic effect of drugs on liver was not observed. $\mathrm{S}+\mathrm{P}$ suppressed hepatic stroma inflammatory infiltration with lymphocytes $(\mathrm{p}<0,05)$. Pathomorphological examination showed marked positive effect of both kinds of treatment on synovia, soft periarticular tissues and cartilage, but it was more expressed under the combined treatment.

Conclusion The obtained results allow to conclude that $S$ alone and its combination with $\mathrm{P}$ had a pronounced antiarthritic effect, showed no toxicity and were well tolerated by animals. These results are in agreement with our previous results, where treatment with $\mathrm{S}$ or $\mathrm{P}$ separately also exerted antiarthritic effect. Pathomorphological examination at the end of the study demonstrated reduced joint swelling in both treated groups, but the combined therapy with $\mathrm{S}+\mathrm{P}$ was more effective.

\section{REFERENCES}

1 Malfait AM, Malik AS, Marinova-Mutafchieva L, et al. The beta 2-adrenergic agonist Salbutamol is a potent supressor of established collagen-induced arthritis: mechanisms of action. J Immunol. 1999;162:6278-83

2 Moller DR, Wysocka M, Greenlee BM, et al. Inhibition of human IL-12 production by pentoxifylline. Immunology 1997;91:197

\section{THU0079 DENDRITIC CELL (DC) SUBSETS IN RHEUMATOID ARTHRITIS (RA) ANALYSED BY 4 COLOUR FLOW CYTOMETRY}

${ }^{1} \mathrm{JL}$ O'Donnell, ${ }^{1} \mathrm{KL}$ Summers, ${ }^{2} \mathrm{~A}$ Rothwell. ${ }^{1}$ Rheumatology, Immunology and Allergy; ${ }^{2}$ Orthopaedic Surgery, Christchurch Hospital, Christchurch, New Zealand

\subsection{6/annrheumdis-2001.956}

Background Distinct myeloid (MDC) and lymphoid (LDC) DC subsets have been described in humans which are thought to regulate the nature and magnitude of the immune response. ${ }^{1} \mathrm{MDC}$ have been associated with a $\mathrm{T}$ helper 1(TH1) mediated response while LDC have been associated with a TH2 mediated response. ${ }^{2}$ Inappropriate regulation of DC function may result in diseases such as RA.

Objectives The composition, co-stimulator molecule expression (CD80, CD86, CD40, 41BB-L) and activation (CD83, CD69) state of these two DC subsets was compared between autologous peripheral blood (PB), synovial fluid (SF) and synovial tissue (ST) from patients with RA undergoing joint replacement surgery. DC were defined as lineage negative (CD3-, CD14-, CD16, CD19- and CD20-) HLA-DR positive cells. MDC were defined as CD11c+ and LDC as CD11c- cells.

Methods Whole blood lysis was used to isolate peripheral blood white cells and size exclusion used to distinguish peripheral blood mononuclear cells (PBMC). Centrifugation was used to isolate cells from freshly drawn SF. ST cells were isolated by mechanical extraction of coarsely minced tissue. Fluorochrome labelled monoclonal antibodies and flow cytometry were used to identify and quantitate cell populations and cell surface proteins of interest. All procedures were carried out either on ice or with cells at $4^{\circ} \mathrm{C}$.

Results In the 5 RA patients peripheral blood DC constituted $0.47 \%$ (mean, range $0.11-1.28$ ) of PBMC. The ratio of MDC to LDC was 1:3.8. Both MDC and LDC expressed weak/low levels of activation and co-stimulator molecules. DC constituted $2.06 \%$ (mean, range $0.78-3.76$ ) of SF mononuclear cells with a ratio of MDC to LDC of 9:1. MDC but not LDC expressed moderate levels of both activation and co-stimulator molecules. ST DCs constituted $8.41 \%$ (mean, range $0.7-22.7$ ) with MDCs making up $100 \%$ of DCs in the 5 samples analysed. These MDC expressed high levels of both activation and co-stimulator molecules. In normal healthy adults $(\mathrm{n}=50)$ DC constituted $1.07 \%$ (mean, median 0.96 , 95\% confidence limits $0.43-2.5$ ) of PBMC with a MDC to LDC ratio of $1: 1 .^{3}$

Conclusion These preliminary results suggest that MDC play an important role in the pathogenesis of joint disease in RA and support the view that RA is predominantly a TH1 mediated disease.

\section{REFERENCES}

1 Banchereau J, et al. Immunobiology of dendritic cells. Annu Rev Immunol. 2000;18:767-811

2 Rissoan MC, et al. Reciprocal control of T helper cell and dendritic cell differentiation. Science 1999;283:1183-6

3 McKenzie J, et al. Personal communication, 2000

\section{THU0080 PROFESSOR}

NF Soroka, NP Mitkovskaya, AB Cheschevik. Internal Diseases, State Medical University, Minsk, Belarus

10.1136/annrheumdis-2001.957

Background Lipid profils in patients with rheumatoid arthritis. Objectives To investigate lipid profiles in patients with active and inactive rheumatoid arthritis (RA) and to assess the relationship of the inflammatory condition of RA with lipid profiles.

Methods Seventy-two patients with RA and 25 age and sex matched healthy controls were studied. Patients with RA had not been treated with corticosteroid drugs prior to the study. Forty patients had active disease, and 32 had inactive disease. Plasma lipoproteins were fractionated by gradient ultracentrifugation into subfractions, and their chemical composition and mass were determined. Total cholesterol, triglyceride, LDL-cholesterol, HDL-cholesterol apolipoprotein AI (apo Al)/apolipoprotein B (apo B), lipoprotein (a) [Lp (a)], lipoprotein-associated plateletactivating factor acetylhydrolase (PAF-AH) activity and C-reactive protein (CRP) were measured in both groups.

Results Patients with active RA had significantly lower plasma total cholesterol and high-density lipoprotein (HDL) cholesterol $(42.9$ vs. $56.1 \mathrm{mg} / \mathrm{dl})$ levels as compared with controls, due to the decrease in the mass of both the HDL2 and HDL3 subfractions. Patients with active RA also had higher plasma triglyceride levels, mainly due to the higher triglyceride content of the very low-density lipoprotein plus the intermediate-density lipoprotein subfraction. The plasma PAF-AH activity in patients with active RA was lower than that in controls, mainly due to the decrease in PAF-AH activity associated with the intermediate and dense low-density lipoprotein subclasses. The levels of apo $\mathrm{Al}$ and HDL-cholesterol were significantly lower in patients than in controls (121.4 vs. $147.4 \mathrm{mg} / \mathrm{dl}$,). The level of Lp (a) was significantly higher in patients than in controls $(29.2$ vs. $19.2 \mathrm{mg} / \mathrm{dl})$. The ratios of apo $\mathrm{B} / \mathrm{apo} \mathrm{Al}$, total cholesterol/HDL-cholesterol, and LDL-cholesterol/HDL-cholesterol were significantly higher in patients than in controls $(0.89$ vs. $0.61,4.6$ vs. $3.2,2.7$ vs. 1.7 , respectively). CRP showed a significant correlation with apo $\mathrm{Al}(\mathrm{r}=-0.47, \mathrm{p}<0.05)$.

Conclusion This study suggests that patients with active RA have altered lipoprotein and apolipoprotein pattems that may possibly expose them to higher risk of atherosclerosis. The inflammatory condition of RA may affect the metabolism of HDL-cholesterol and apo A1. Our study to show that patients with active RA exhibit low levels of HDL2 and HDL3 and are deficient in 